\title{
Avaliação da eficácia da aplicação das folhas da ochroma pyramidale na concentração gravítica do ouro na sub-bacia do Alto Revuè
}

\author{
Evaluation of the effectiveness of the application of ochroma pyramidale leaves in the gravity \\ concentration of gold in the Alto Revuè sub-basin
}

Evaluación de la efectividad de la aplicación de hojas de ochroma pyramidale en la concentración gravitacional de oro en la subcuenca del Alto Revuè

\begin{abstract}
Resumo
À nível mundial, a amalgamação do ouro é convencionalmente feita com recurso ao mercúrio. Porém, a poluição resultante da utilização do mercúrio é das mais preocupantes, dado que tem efeitos devastadores na saúde humana como no meio ambiente. Assim sendo, esta pesquisa teve como objetivo avaliar a eficácia da aplicação das folhas da Ochroma pyramidale no apoio da concentração gravítica do ouro na sub-bacia do alto Revuè, no distrito de Manica, no extremo Centro-Oeste da província de Manica, região Centro de Moçambique. Para tal, o estudo baseou-se na pesquisa bibliográfica e documental e na testagem do processo do apoio da concentração gravítica do ouro com recurso às folhas desta planta. Foram feitas 10 testagens utilizando a folha desta planta, onde participaram 251 mineradores. De acordo com os resultados, as folhas da Ochroma pyramidale demonstraram ser capazes de clarear e separar o ouro dos outros materiais apenas em áreas onde as dimensões das partículas do ouro eram relativamente maiores. No entanto, deste trabalho verificou-se que há estudos que comprovam que a Manhiot esculenta Grantz (mandioca amarga) é eficaz no apoio da concentração gravítica do ouro, atraindo as partículas do ouro, o que marca o início de um futuro muito promissor na mineração pois, a mandioca amarga já se pode utilizar, substituindo o mercúrio. Com isso, recomenda-se a realização de trabalhos-piloto de demonstração da utilização da mandioca amarga.
\end{abstract}

Palavras-chave: Amalgamação; Concentração gravítica; Ochroma pyramidale; Plantas cianogênicas.

\begin{abstract}
Worldwide, gold amalgamation is conventionally done using mercury. However, the pollution resulting from the use of mercury is of the most concern, as it has devastating effects on human health and the environment. This work addresses the evaluation of the effectiveness of the application of Ochroma pyramidale leaves to support the gravity concentration of gold in the Alto Revuè sub-basin, in the district of Manica, in the extreme center-west of the province of Manica, in the center of Mozambique. The work was essentially based on bibliographical and documental research and on testing the process of supporting the gravity concentration of gold using the leaves of the plant. 10 tests were carried out using the leaf of this plant, in which 251 miners participated. From the tests carried out, it was observed that the leaves of Ochroma pyramidale proved to be able to lighten and separate the gold from other materials only in areas where the dimensions of the gold particles were relatively larger. However, there are studies that prove that Manhiot esculenta Grantz (bitter cassava) is effective in supporting the gravity concentration of gold, attracting gold particles, which marks the beginning of a very promising future in mining because bitter cassava can already be used, replacing the mercury. Therefore, it is recommended to carry out pilot work for demonstrating the use of bitter cassava.
\end{abstract}

Keywords: Amalgamation; Gravity concentration; Ochroma pyramidale; Cyanogenic plants. 


\begin{abstract}
Resumen
En todo el mundo, la fusión de oro se realiza convencionalmente utilizando mercurio. Sin embargo, la contaminación resultante del uso de mercurio es la más preocupante, ya que tiene efectos devastadores sobre la salud humana y el medio ambiente. Por lo tanto, esta investigación tuvo como objetivo evaluar la efectividad de la aplicación de hojas de Ochroma pyramidale para soportar la concentración gravitacional de oro en la subcuenca del Alto Revuè, en el distrito de Manica, en el extremo Centro-Oeste de la provincia de Manica, región central de Mozambique. Para ello, el estudio se basó en investigaciones bibliográficas y documentales y en probar el proceso de soporte de la concentración gravitacional de oro utilizando las hojas de la planta. Se realizaron 10 pruebas utilizando la hoja de esta planta, en las que participaron 251 mineros. Según los resultados, las láminas de Ochroma pyramidale demostraron ser capaces de limpiar y separar el oro de otros materiales solo en áreas donde el tamaño de las partículas de oro era relativamente mayor. Sin embargo, a partir de este trabajo se encontró que existen estudios que prueban que la Manhiot esculenta Grantz (yuca amarga) es eficaz en sustentar la concentración gravitacional del oro, atrayendo las partículas de oro, lo que marca el inicio de un futuro muy prometedor en la minería porque la yuca amarga ahora puede ser usado, reemplazando el mercurio. Por tanto, se recomienda realizar trabajo piloto para demostrar el uso de mandioca amarga. Palabras clave: Fusión; Concentración por gravedad; Ochroma pyramidale; Plantas cianogénicas.
\end{abstract}

\title{
1. Introdução
}

À nível mundial, a amalgamação ${ }^{1}$ do ouro é convencionalmente feita com recurso ao mercúrio (Hg) (Veiga, AngelociSantos \& Meech, 2014). Porém, a poluição resultante da utilização do mercúrio é das mais preocupantes, dado que tem efeitos devastadores locais, regionais e globais tanto na saúde humana como no meio ambiente. Estima-se que a quantidade de mercúrio exposta anualmente ao meio ambiente seja de mais de 2.000 toneladas (Torkaman et al., 2021).

Nas regiões Centro e Norte de Moçambique, a quantidade de mercúrio exposta ao meio ambiente também é preocupante (Fritz, Mcquilken, Collins \& Weldegiorgis, 2018). A província de Manica e, sobretudo, a sub-bacia do Alto Revuè, situada no distrito de Manica, é uma das mais afectadas (Veiga et al., 2005).

A contínua emissão e poluição do meio ambiente pelo mercúrio leva ao agravamento da sua transformação e interação com outros diversos elementos do meio ambiente, tornando-o potencialmente nocivo (Dondeyne et al., 2007; Homrich, Fernandes \& Viera, 2014). É muito perigoso para as pessoas diretamente envolvidas na mineração de ouro, devido a exposição à inalação directa ${ }^{2}$. As pessoas que não estão diretamente envolvidas na amalgamação do ouro podem ser contaminadas por via da cadeia alimentar e por inalação do mercúrio exposto na atmosfera nas proximidades das áreas de processamento do ouro com mercúrio pois, o mercúrio facilmente pode se deslocar para áreas próximas da mineração através do vento (Veiga et al., 2005).

Para a prevenção dos impactos ambientais e socioeconómicos negativos resultantes da utilização do mercúrio é fundamental que sejam adotadas práticas sustentáveis de mineração do ouro (Castigo et al. 2018). Apesar da área da floresta de Chocó, na Colômbia, ter experiências ancestrais de concentração gravítica do ouro com recurso às folhas da Ochroma pyramidale, planta vulgarmente conhecida por pau-de-balsa, pau-de-lebre, pau da jangada ou por balsa, da família Malvaceae, nativa da Amazónia Ocidental (Sinergia Ambiental Gaia Viva [SAGV], 2018), até então, a amalgamação do ouro é essencialmente feita com recurso ao mercúrio (Torkaman et al., 2021).

Nesta perspectiva, este trabalho teve como objetivo avaliar a eficácia da aplicação das folhas da Ochroma pyramidale no apoio da concentração gravítica do ouro ${ }^{3}$, na sub-bacia do Alto Revuè, situado no distrito de Manica, província de Manica,

\footnotetext{
${ }^{1}$ Quando o minério é extraído, o ouro obtém-se com areia e outros minerais como o ferro. Para a sua separação da areia e dos outros metais menos denso do ouro em pó, após a lavagem da mistura, numa rampa com pano de feltro, utiliza-se o Hg. Ao processo de separação das partículas do ouro dos outros materiais utilizando um metal é que se designa de amalgamação (Dondeyne et al., 2007).

${ }^{2}$ De acordo com aMedicus Mundi e Centro Terra Viva (MM \& CTV, 2018), a contaminação pelo mercúrio causa enormes problemas de saúde. Cria problemas como de perda de memória, tremor, pneumonia, gengivite, tremores, depressão, nervosismo, falha renal, cardiovasculares, perfuração gástrica, esofágica, demência e em casos graves pode levar à morte.

${ }^{3}$ Segundo Lins (1992), à concentração gravítica refere-se ao processo no qual partículas de diferentes densidades, tamanhos e formas se separam pela ação da força de gravidade ou pela força centrífuda.
} 
na região Centro de Moçambique. O presente trabalho pode contribuir para a melhor tomada de decisão sobre as medidas de prevenção dos impactos ambientais e socioeconómicos negativos decorrentes da amalgamação do ouro. $\mathrm{O}$ apoio da concentração gravítica do ouro através das folhas da Ochroma pyramidale e a redução da utilização do mercúrio pode garantir a qualidade da água e a conservação da biodiversidade. Com a tomada de medidas de prevenção da degradação ambiental e de riscos à saúde, o estudo pode contribuir para o bem-estar das famílias locais.

\section{Metodologia}

Realizou-se um estudo exploratório, envolvendo o levantamento bibliográfico e entrevistas à geólogos experientes na mineração do ouro. De acordo com Zanella (2013), a pesquisa exploratória tem a finalidade de obter maior conhecimento sobre um determinado fenômeno ou problema.

A pesquisa caracteriza-se por uma abordagem qualitativa. Segundo Guerra (2014), a pesquisa qualitativa procura compreender um determinado fenômeno que se estuda, em seu ambiente, interpretando-o de acordo com a perspectiva do próprio sujeito, sem se preocupar com a representatividade númerica.

Quanto aos procedimentos, trata-se de uma pesquisa experimental. De acordo com Petry (2013), a pesquisa experimental envolve reprodução de fenômenos, dentro de um plano de modificações sistemáticas das variáveis independentes, em relação à um certo evento, com o fim de descobrir as condições antecedentes responsáveis pelos eventos subsequentes. Para Gerhardt e Silveira (2009), a pesquisa experimental pode ser feita no laboratório ou no campo.

No entanto, esta foi realizada no campo, com a participação dos mineradores artesanais e de pequena escala. Para a análise da eficácia das folhas da Ochroma pyramidale, na sub-bacia do Alto Revuè, foram feitas 10 testagens de aplicação das folhas da planta no apoio da concentração gravítica do ouro. As testagens foram feitas nas comunidades de Chinhagore, Maridza, Mucudo, Chua, Matoraburo, Chimedza, Mudza, Josina Machel, Fenda e Mutsintsa. A comunidade de Chinhagore, com excepção das outras comunidades, onde extraem ouro aluvionar, processa ouro das rochas.

Para a revisão bibliográfica foram utilizados diversos artigos científicos e livros publicados por meios impressos e electrónicos. Os artigos utilizados foram pesquisados no Google Scholar.

\section{Resultados e Discussão}

\subsection{Apoio da concentração gravítica do ouro através das folhas da ochroma pyramidale}

O processo de apoio à concentração gravítica iniciou com a obtenção do material tratado, contendo partículas de ouro, areia e outros materiais como o ferro. Para separar o ouro da areia e do outro material, com uma pequena quantidade de água (cerca de $500 \mathrm{ml}$ ), friccionou-se com pressão uma folha triturada da Ochroma pyramidale numa bacia, por cerca de dois à três minutos, onde passado este período surgia um líquido verde com espuma (Figura 1). 
Figura 1. Líquido verde resultante da fricção das folhas da planta.

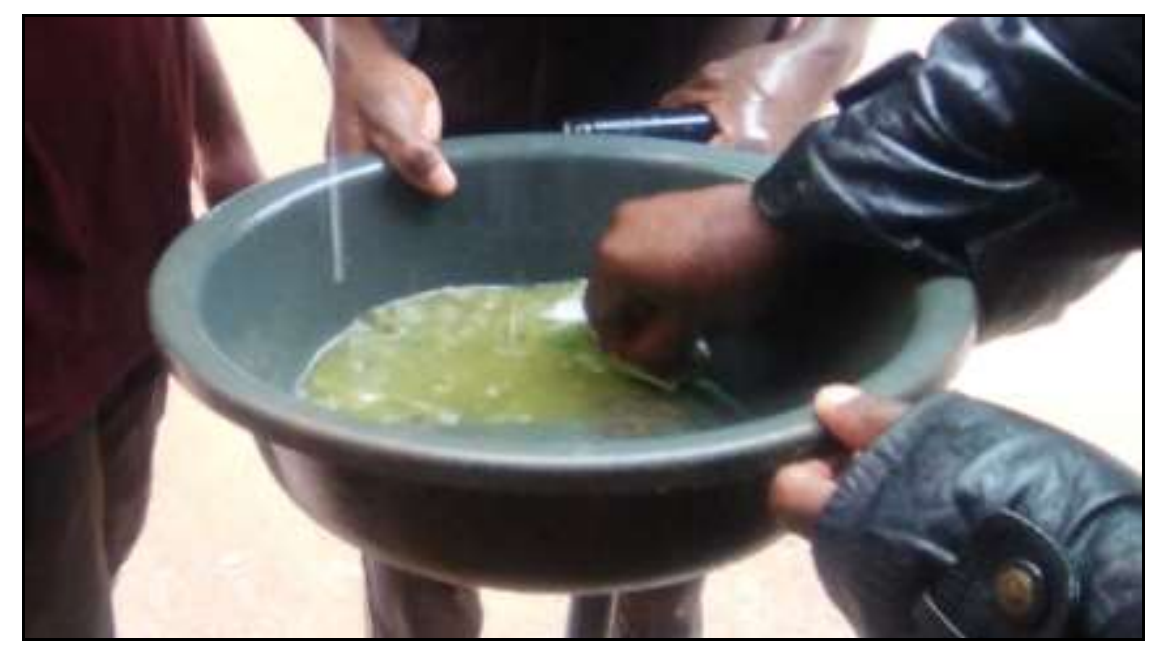

Fonte: Autores (2021).

A seguir, a mistura do líquido da planta, ouro, areia e outro material foi se mexendo por mais um período de cerca de cinco minutos. Passado este tempo, à esta mistura adicionou-se água limpa para o clareamento dos líquidos. Com este processo, o ouro aluvionar, com partículas relativamente maiores visualizava-se, separado da areia e de outros minerais (Figura 2).

Figura 2. Visualização da separação do ouro de outros materiais.

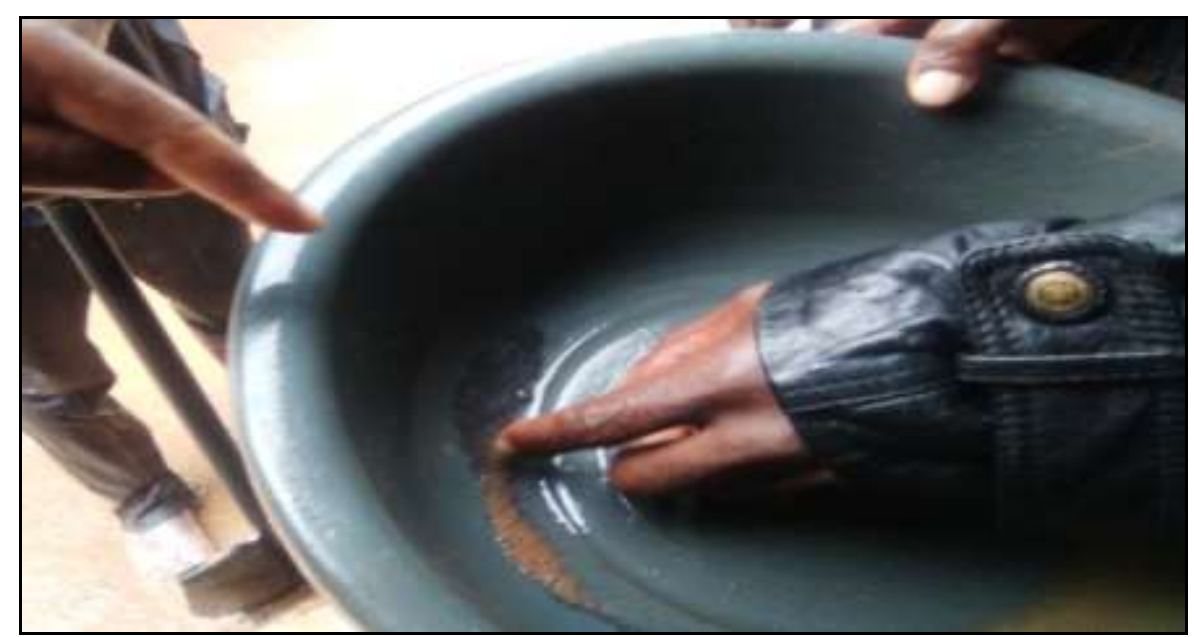

Fonte: Autores (2021).

Da análise feita do resultado obtido constatava-se que o ouro apenas clareou-se e separou-se do outro material como a areia ou o ferro. Portanto, as partículas do ouro não se contraíram. Contudo, para o caso onde o ouro era de dimensões relativamente maiores, de acordo com os mineradores envolvidos nas testagens, o nível de separação com os outros materiais foi satisfatório.

De acordo com a entrevista concedida ao Professor Doutor Veiga, em Maio de 2021, o clareamento do ouro se dá pela planta possuir algum detergente que limpa as substâncias húmicas da superfície do ouro. O suco não dissolve o ouro e nem produz uma resina que atrai partícolas muio finas [...], apenas dispersa as particulas permitindo maior facilidade para a 
concentração gravítica do ouro. Carrisso e Correia (2004) e Lins (2010), referem que a separação ocorrida deve-se à diferença de densidades do ouro e outros materiais.

Porém, em áreas com partículas de ouro com dimensões relativamente mais finas (minério ${ }^{4}$ moído ou em argila e silte), o ouro não se distinguiu de outros materiais. Esta situação ocorreu na comunidade de Matoraburo, onde o ouro extrai-se nas margens do rio Chimedza, num local onde predomina argila e silte. Igualmente ocorreu na comunidade de Chinhagore, onde processa-se ouro das rochas, que quando são moídas, obtém-se partículas muito finas.

Com isso, pode-se depreender que com o suco da balsa as partículas de ouro com dimensões relativamente maiores apenas clareiam-se e separam-se de outros materiais.

Com isso, o maior desafio que se impõe é da testagem do apoio da concentração gravítica com recurso à outras plantas. De acordo com a Organização Mundial da Saúde (OMS, 1993) e Torkaman, Veiga, Lima, Oliveira e Lavkulich (2019), existem mais de 2,000 plantas cianogênicas 5 . Torkaman et al. (2019), dentre outras, menciona as ilustradas na Tabela 1.

Tabela 1. Plantas cianogências e respetivo nível de concentração.

\begin{tabular}{ccc}
\hline Nome científico & Nome vulgar & $\begin{array}{c}\text { Nível de concentração do cianeto (em } \\
\mathbf{m g / K g ~ o u ~ m g / l i t r o ) ~}\end{array}$ \\
\hline Bambusoideae & Pontas dos brotos imaturos dos bambús & 7,700 \\
Phaseolus lunatus & Feijão-de-lima claro & 3,000 \\
Phaseolus lunatus & Feijão-de-lima escuro do Porto Rico & 2,900 \\
Manhiot esculenta & Raízes secas da mandioca amarga & 2,360 \\
Manhiot esculenta & Raízes frescas da mandioca amarga & 380 \\
\hline
\end{tabular}

Fonte: Torkaman et al. (2019).

De acordo com Torkaman et al. (2021), dos testes de apoio à concentração gravítica feitos com recurso às raízes da mandioca amarga, provou-se a sua eficácia. Ao invés de apenas clarear e dispersar as partícolas do ouro, as partículas de ouro, incluíndo de dimensões menores, atraem-nas por ter cianeto. Torkaman et al. (2021), refere ainda que o cianeto das plantas, contrariamente ao mercúrio, não persiste no meio ambiente pois, os processos físicos, químicos e biológicos naturais o transformam em produtos menos tóxicos.

No entanto, como pode-se observar na Tabela 1, há outras plantas como o bamboo que possuem níveis mais elevados de cianeto que a mandioca amarga. Deste modo, ainda podem ser realizados estudos comparativos de níveis de eficácia das plantas cianogênicas no apoio da concentração gravítica.

\section{Conclusões e Sugestões}

As folhas da Ochroma pyramidale demonstraram ser capazes de clarear e auxiliar na separação do ouro dos outros materiais apenas em áreas onde as dimensões das partículas do ouro eram relativamente maiores e em áreas onde eram de dimensões muito menores o resultado não foi satisfatório.

Dos resultados desta pesquisa verificou-se que há um estudo que comprova que a mandioca amarga é eficaz no apoio da concentração gravítica do ouro, atraindo as partículas do ouro, o que marca o início de um futuro muito promissor.

${ }^{4}$ De acordo com Da Luz e Lins (2010), ao minério refere-se à qualquer rocha constituída de um mineral ou agregado de minerais, contendo um ou mais minerais valiosos.

${ }^{5}$ De acordo com Tokarnia, Döbereiner, Peixoto, Brito e Barbosa (2012), consideram-se plantas cianogênicas as que possuem glicosídeos cianogênicos, que quando são lidrolisados libertam cianeto, ou seja, ácido cianídrico (HCN). 
De acordo com Torkaman et al. (2021), com base nos resultados satisfatórios alcançados, já se pode utilizar a mandioca amarga, substituindo o mercúrio.

Sugere-se a realização de trabalhos-piloto da demonstração da utilização da mandioca amarga e, considerando que o bambú possui níveis de cianeto muito elevados que da mandioca, poderão se realizar experiências de utilização desta planta para a substituição do mercúrio e outros metais pesados.

\section{Agradecimentos}

Especial agradecimento vai ao Marcello M. Veiga P. Eng., PhD, da Universidade de British Columbia, de Canadá, pelo apoio e observações dadas; agradece-se ainda a todos os dirigentes e funcionários da Agência Nacional para o Controlo da Qualidade Ambiental (AQUA), pelas valiosas contribuições e apoios dados; ao Dr. Calisto Manuel Máquina, da Universidade Zambeze, pelo seu grande apoio e a todos os que, directa ou indirectamente, contribuíram para esta realização, o nosso muito obrigado.

\section{Referências}

Carrisso, R. C. \& Correia, J. C. G. (2004). Classificação e peneiramento. Comunicação Técnica Elaborada para o Tratamento de Minérios. Centro de tecnologia mineral. (4a ed.), 5. $197-238$.

Castigo, P., Fopenze, R., Ferrão, C., Nhaca, F., Charles, F., Júlio, A. \& Estevão, D. (2018). Avaliação dos impactos ambientais da mineração do ouro no distrito de Manica: Estudo de caso da Sub-bacia do Alto Revuè. Chimoio

Da Luz, A. B. \& Lins, F.A. F. (2010). Introdução ao tratamento de minérios. Comunicação Técnica Elaborada para o Tratamento de Minérios. Centro de tecnologia mineral. (5a ed.), 1, $3-20$.

Dondeyne, S., Ndunguro, E., Ferrão, C., Jantar, P., Rafael, P., \& Nhaca. F. (2007). Em busca de ouro: Garimpo e desenvolvimento sustentável uma difícil reconciliação. Chimoio

Fritz, M., Mcquilken, J., Collins, N. \& Weldegiorgis, F. (2018). Global Trends in Artisanal and Small-Scale Mining (ASM): A review of key numbers and issues. Winnipeg. International Institute of Sustainable Development.

Guerra, E. (2014). Manual de pesquisa qualitativa.

Homrich, B. S., Fernandes, C. R P. \& Viera, J. R. G. (2014). O potencial tóxico do mercúrio e os impactos da gestão inadequada de seus resíduos ao meio ambiente e à saude. V Congresso Brasileiro de Gestão Ambiental. Belo Horizonte/MG - 24 a 27/11/2014

Lins, F. F. (1992). Aspectos da garimpagem do ouro.

Lins, F. F. (2010). Concentração gravítica. Centro de Tecnologia Mineral. Comunicação Técnica Elaborada para o Tratamento de Minérios, (5a ed.), 7. 301 326.

Medicus Mundi e Centro Terra Viva (MM \& CTV, 2018). Projecto mineração artesanal: direitos ambientais e culturais em Cabo-Delgado.

Gerhardt, T. E. \& Silveira, D. T. (2009). Métodos de pesquisa. UFRGS

Organização Mundial da Saúde (OMS, 1993). Cyanogenic glycosides: Toxicological evaluation of certain food additives and naturally occurring toxicants. Geneva. WHO Food Additive Series 30. httpDZA்ȦwwwžinchemžorgA்documents $\dot{j} j e c f a A ̇ j e c m o n o \dot{A}$ v30je18žhtm.

Petry, G. A. (2013). Ciência aplicada e experimentos científicos. Instituto Federal de Santa Catarina

Sinergia Ambiental Gaia Viva (SAGV, 2018). Planta substitui o mercúrio na mineração de ouro. Vídeo de duração de 5:45 mn. https://www.youtube.com/watch?v=XKG-Q3Dvdzk.

Tokarnia, C., Döbereiner, J., Peixoto, P. V., Brito, M., \& Barbosa, J. (2012). Plantas tóxicas do Brasil para animais de produção. Editora Heianthus, (2a ed.), 443-459

Torkaman, P., Veiga, M. M., Lima, L.R.P. Oliveira, L. A., \& Lavkulich L. M. (2019). Utilization of cyanogenic plants to extract gold. Canada. University of British Columbia.

Torkaman, P., Veiga, M. M., De Andrade Lima, L. R. P., Oliveira, L. A., Motta, J. S., Jesus, J. L., \& Lavkulich L. M. (2021). Leaching gold with cassava: an option to eliminate mercury use in artisanal gold mining. Canada

Veiga, M. M., \& Angeloci-Santos, M. (2014). Review of barriers to reduce mercury use in artisanal gold mining. In: The Extractive Industries and Society $351-361$.

Veiga, M., Shoko, D., Spiegel, S., Savornin, O., Rafael, P., Castigo, P., N'dunguru, E., Sandramo, R. \& Jantar, P. (2005). Pilot project for the reduction of mercury contamination resulting from artisanal gold mining fields in the Manica district of Mozambique

Zanella, L. C. H. (2013). Metodologias de pesquisa. (2a ed.), UFSC 